stellungsbeauftragten ein politisches, so dass man in der Diskussion zumeist nicht als Juristin, sondern als Person hinter der Argumentation stehen muss. Ich merke auch, dass ich auf Ungerechtigkeiten im Gleichstellungsbereich doch mehr erstaunt oder ärgerlich reagiere als ich es in „traditionellen“ Rechtsstreitigkeiten je getan hätte. Daher ist eine ausgeprägte feministische Grundeinstellung unabdingbar; auch wenn Mitarbeitende - insbesondere der Jura-Fakultät - auf diese Einstellung und auch auf den Arbeitsschwerpunkt nicht immer wohlwollend reagieren.

Daneben musste ich zugegebenermaßen erst einmal lernen, andere als juristische Kenntnisse und Fähigkeiten als Kompetenzen anzuerkennen. Häufig hatte ich in den ersten Wochen das Gefühl, dass ich nur etwas geleistet hatte, wenn ich mit Gesetzen oder Urteilen gearbeitet hatte. Die Konzipierung eines Mentoring-Programmes oder die Evaluierung bestimmter Karriereverläufe haben grundsätzlich nichts mit dem zu tun, was im Studium oder Referendariat gelernt wird. Ich gebe es ungern zu, aber einige Male kam das Gefühl auf, meine Ausbildung zu verschwenden und diese ganze Arbeit auch mit einem weniger anstrengenden Studium erledigen zu können. Gerade in der interdisziplinären Arbeit ist dies eine despektierliche und auch ungerechtfertigte Auffassung gegenüber anderen Fachgebieten. Dennoch stellt sich diese Frage wahrscheinlich mehreren Frauen, die direkt nach dem Examen in einen alternativen juristischen Beruf einsteigen und viel nicht-juristische Arbeit zu erledigen haben. Erst mit der Zeit verbuchte ich diese Tätigkeiten unter akademische Arbeit, die im - wenn auch mittelbaren - Zusammenhang mit meiner Ausbildung steht. Dennoch freue ich mich noch immer über jeden Verwaltungsakt, der auf meinem Tisch landet, und über jede Diskussion, die ich mit anderen Jurist(inn)en führen kann. Aber auch die Erkenntnisse der Geschlechterforschung sind in der Gleichstellungsarbeit regelmäßig zu verteidigen und die Aus- legung von „Gender“ und „Gleichstellung“ mindestens genauso vielfältig wie die von „Treu und Glauben“. Da der universitäre Gleichstellungsprozess zunehmend von dem Streit um Fördermittel, Rankings und Kompetenzlosigkeit determiniert wird, müssen sich gerade die Gleichstellungsbeauftragten mit ihrem gesetzlichen Auftrag, aber auch mit Hilfe der Geschlechterforschung behaupten.

Es dauerte zudem auch mehr als drei Monate, bis ich außerhalb des Gleichstellungsbüros als selbstständige Ansprechpartnerin wahrgenommen wurde. Dafür lief es dann richtig gut: Einladungen zu Vorträgen, Anfragen für Gutachten und Stellungnahmen, Einladungen zu Arbeitsgemeinschaften, Mitarbeit in Netzwerken. Juristische Kompetenzen sind in der Gleichstellungsarbeit gefragt und geschätzt; ohne einen wissenschaftlichen Bezug zur Geschlechterforschung und Genderkompetenz kommt man in der praktischen Gleichstellungsarbeit allerdings nicht weiter. Inhaltlich ist es eine sehr anspruchsvolle und spannende Arbeit. Jedoch muss ich mich mit den prekären Arbeitsbedingungen im Wissenschaftsbetrieb auseinandersetzen, die im Gleichstellungsbereich noch um einiges schlechter sind: innerhalb von acht Monaten unterschrieb ich (zumeist in letzter Sekunde) viermal einen weiteren befristeten Arbeitsvertrag. Erst seitdem habe ich eine Stelle, die zumindest aus einem 5-Jahres-Programm finanziert wird. Zudem wird oft die Arbeitsweise einer Wissenschaftlerin erwartet, die eine Professur anstrebt; dass das Mensch-Sein für manche erst mit W3 beginnt, stellte sich nicht nur als blöder Spruch heraus; Überstunden sind die Regel und erst, nachdem ich mich neben meiner Vollzeitstelle zur Promotion entschlossen habe, lässt sich für mich die reguläre Arbeitszeit einigermaßen einhalten. Dennoch werde ich im Gleichstellungsbereich weiter arbeiten, denn eine bessere Ausschöpfung meiner Kompetenzen und Überzeugungen ist schwer vorstellbar.

\title{
Vereinbarkeit von Kind und Karriere bei der Justiz
}

\section{Dorothea Gaudernack \\ Staatsanwältin, Augsburg}

„Das war sicherlich ein Unfall, oder?“ So der Kommentar meiner Vorgesetzten nach der Geburt meines ersten Sohnes im Jahr 2006. Ein Unfall? Nein: ich war fast 30 Jahre alt, seit einiger Zeit verheiratet und, ich gestehe: Ja! Ich hatte einen Kinderwunsch!

Als ich mit 18 das Jurastudium begonnen hatte, sah ich mich noch als Diplomatin beim Auswärtigen Amt oder im Chanel-Kostüm für wichtige Vertragsverhandlungen um die Welt jetten. Kinderwunsch? Fehlanzeige! Im Studium lernte ich jedoch den Mann kennen, mit dem ich mir vorstellen konnte, Kinder zu haben. Während meiner Referendarstationen bei zwei internationalen Großkanzleien und bei der Bot- schaft in Rom kam die Ernüchterung und die Erkenntnis, dass die wenigen Frauen mit interessanten Positionen meist keine Kinder hatten. Meine männlichen Ausbilder übrigens schon, aber ihre Frauen waren entweder nicht berufstätig oder im öffentlichen Dienst.

Nach dem Referendariat entschied ich mich also bewusst für die Justiz, weil ich davon ausging, dass sich dort „Kind und Karriere" am besten verwirklichen ließen. Nachdem ich ca. eineinhalb Jahre als Proberichterin tätig war, bekam ich keineswegs ungeplant - meinen ersten Sohn. Als er zehn Monate alt war, fing ich mit zwei Drittel der regulären Arbeitszeit wieder an zu arbeiten, allerdings bei einem anderen Gericht, in einem ganz anderen Rechtsgebiet. Obwohl mein Sohn sich in der Krippe wohl fühlte und ich mich darüber freute, meinen Verstand wieder zu betätigen und nicht mehr nur mit anderen 
Müttern über Windeln, Beikost und Co. zu sprechen, fiel mir der Wiedereinstieg schwerer als erwartet: das „Abgeben“ in der Krippe war oft mit Tränen verbunden; mein Sohn, der zu diesem Zeitpunkt ja noch ein Baby war, schleppte einen Infekt nach dem anderen an und hatte Phasen, in denen er nachts stündlich aufwachte. Mein Mann (übrigens Rechtsanwalt) arbeitete von morgens bis abends, meine Freundinnen waren alle berufstätig und kinderlos oder wohnten weit weg. Ich war dauermüde, erschöpft und fühlte mich wie ein Hamster im Rad. Für mich selbst, für Freizeitaktivitäten, Sport, einen Friseurbesuch oder für uns als Paar blieb überhaupt keine Zeit. Abends schlief ich oft über den mitgeschleppten Akten ein.

Völlig unerwartet wurde mir dann angeboten, in mein Heimatbundesland zu wechseln. Nachdem sich dort auch eine attraktive Jobchance für meinen Mann bot, packten wir kurzentschlossen die Gelegenheit beim Schopfe, kauften binnen weniger Tage ein Einfamilienhaus mit Garten und tauschten unser cooles, aber anstrengendes urbanes Leben gegen die bodenständige Idylle der bayerischen Kleinstadt, in der meine Eltern leben. Wenn mir das vor wenigen Jahren jemand prophezeit hätte, hätte ich ihn vermutlich für vollkommen verrückt erklärt! Zurück in der Heimat konnte ich dank der Hilfe meiner Eltern bei der Kinderbetreuung sogar eine Vollzeitstelle bei Gericht annehmen. Vor gut einem Jahr habe ich - schon wieder vorsätzlich meinen zweiten Sohn bekommen, und seit seinem ersten Geburtstag bin ich, zur Zeit als Staatsanwältin, wieder berufstätig - wegen des weiten Arbeitsweges im Moment nur zu 50 Prozent.

Ich wollte Richterin werden, weil ich der Ansicht war, in der Justiz ließen sich „Kind und Karriere“ am besten vereinbaren. Heute, fünf Jahre und zwei Kinder später, bin ich mir diesbezüglich nicht mehr so sicher: „Kind und Beruf“ lässt sich vermutlich in der Justiz oft einfacher verwirklichen als etwa bei Anwältinnen oder Juristinnen in Unternehmen: Teilzeitarbeit ist in der Regel kein Problem. Als Richterin hat man keine festen Arbeitszeiten, kann, wenn man nicht gerade Sitzung hat, schnell mal weg, wenn die Kita anruft und das Kind krank ist, und die Freiheit des Richterberufs erlaubt es auch, einen Teil der Arbeit abends zu
Hause zu erledigen. Aber „Kind und Karriere“ ist auch in der Justiz nicht einfach: die Justiz ist weiblich geworden - aber eben vor allem im sogenannten Eingangsamt, d. h. im R1-Bereich. Bei den Beförderungsstellen sind Frauen insgesamt immer noch unterrepräsentiert, und die wenigen Vorzeigefrauen in der Justiz haben häufig keine Kinder. Über die Gründe dafür kann ich nur mutmaßen. Es mag daran liegen, dass es für Mütter einfach schwer ist, die für eine Beförderung erforderlichen Anforderungen an Mobilität und Flexibilität zu erfüllen: So ist z. B. eine Abordnung an das Bundesjustizministerium oder gar nach Brüssel für eine Mutter einfach nicht zu leisten - es sei denn, der dazugehörige Mann ist freischaffender Künstler oder sonst in keiner Weise beruflich gebunden. Es mag auch daran liegen, dass viele Mütter in den für das berufliche Fortkommen entscheidenden Jahren nur Teilzeit arbeiten und bestimmte „karriereträchtige“ Positionen, z. B. Referentenstellen im Ministerium, schon faktisch nicht in Betracht kommen. Durchaus verbreitet scheint auch die Ansicht zu sein, Mütter gäben ihren beruflichen Ehrgeiz grundsätzlich an der Kreißsaaltür ab. Es mag auch Frauen geben, für die sich die Prioritäten nach der Geburt eines Kindes tatsächlich so stark verändern, dass der Beruf allenfalls eine untergeordnete Rolle spielt. Aber auch wenn meine „Buben“ das Beste und Wichtigste in meinem Leben sind, hat es mir persönlich jedenfalls nie gereicht, nur „die Mama von“ zu sein (die Unkenntnis des mütterlichen $\mathrm{Na}$ mens ist ein in Krabbelgruppen verbreitetes Phänomen). Ich liebe meinen Beruf und gehe gerne arbeiten - auch wenn ich nicht selten mit „Verdient dein Mann nicht genug?“ oder einem „Muss der Kleine wirklich schon in die Krippe? “ konfrontiert werde.

Mein persönliches Fazit: trotz des „Rabenmutter-Images“ (angeblich soll es diesen Begriff in anderen Sprachen ja so gar nicht geben), obwohl der Spagat zwischen Gerichtssaal und Krippe, zwischen Akten und Lego anstrengend ist und ohne die Unterstützung der Familie bzw. eines sonstigen sozialen Netzwerks und eine ausgeklügelte Organisation nicht funktionieren würde, würde ich die Entscheidung für Kinder und Beruf immer wieder so treffen! 